\title{
Thermal Jump Effects on Boundary Layer Flow of a Jeffrey Fluid Near the Stagnation Point on a Stretching/Shrinking Sheet with Variable Thermal Conductivity
}

\author{
M. A. A. Hamad, ${ }^{1}$ S. M. AbdEl-Gaied, ${ }^{1}$ and W. A. Khan ${ }^{2}$ \\ ${ }^{1}$ Mathematics Department, Faculty of Science, Assiut University, Assiut 71516, Egypt \\ ${ }^{2}$ Department of Engineering Sciences, PN Engineering College, National University of Science, Pakistan \\ Correspondence should be addressed to S. M. AbdEl-Gaied; sagaied123@gmail.com
}

Received 27 June 2013; Accepted 29 October 2013

Academic Editor: Boming Yu

Copyright (c) 2013 M. A. A. Hamad et al. This is an open access article distributed under the Creative Commons Attribution License, which permits unrestricted use, distribution, and reproduction in any medium, provided the original work is properly cited.

\begin{abstract}
A mathematical model will be analyzed in order to study the effects of thermal jump and variable thermal conductivity on flow and heat transfer near the stagnation point on a stretching/shrinking sheet in a Jeffrey fluid. The highly nonlinear partial differential equation of Jeffrey fluid flow along with the energy equation are transformed to an ordinary system using nondimensional transformations. The arising equations are solved for temperature, velocity, shear stress, and heat flux using finite difference method. The effect of the influences parameters is discussed. For nonradiation regular viscous fluid our results are as that by Nazar et al. (2002).
\end{abstract}

\section{Introduction}

It is well known that the thermophysical properties of a fluid play an important role in the engineering applications in aerodynamics, geothermal systems, crude oil extractions, ground water pollution, thermal insulation, heat exchanger, storage of nuclear waste, and so forth, convective flows over bodies. The change in the thermal conductivity with temperature is an important property [1-5]. Prasad and Vajravelu [6] investigated the effect of variable thermal conductivity in a nonisothermal sheet stretching through power law fluids while Prasad et al. [4] reported similar studies for viscoelastic fluids. Abel et al. [7] studied combined effects of thermal buoyancy and variable thermal conductivity on a magnetohydrodynamic flow and the associated heat transfer in a powerlaw fluid past a vertical stretching sheet in the presence of a nonuniform heat source. The general findings of these studies were that the effects of variable thermal conductivity increase the shear stress. The temperature at wall increase with an increase in variable thermal conductivity by Seddeek et al. [8] . Prasad et al. [9] found that the variable thermal conductivity has an impact in enhancing the skin friction coefficient; hence, fluids with less thermal conductivity may be opted for effective cooling. Abel et al. [10] concluded that the variable thermal conductivity increases the temperature distribution in both prescribed surface temperature and prescribed heat flux cases. Mahanti and Gaur [11] investigated the effects of linearly varying viscosity and thermal conductivity on steady free convective flow of a viscous incompressible fluid along an isothermal vertical plate in the presence of heat sink. Deissler [12] obtained that the effects of second-order terms on the velocity and temperature jumps at a wall are by a physical derivation. The analysis used the concepts of effective mean free paths for momentum and energy transfer; the effective mean free paths are obtained from known viscosities and thermal conductivities. Rahman and Eltayeb [13] studied numerically the convective slip flow of slightly rarefied fluids over a wedge with thermal jump and temperature dependent transport properties such as fluid viscosity and thermal conductivity. Cipolla Jr. [14] studied the temperature jump in polyatomic gas, also Kao [15] and Latyshev and Yushkanov [16] studied the temperature jump. The flow and heat transfer of Jeffry fluid near stagnation point on a stretching/shrinking sheet with parallel external 
flow was investigated by Turkyilmazoglu and Pop [17]; Akram and Nadeem [18] discussed the peristaltic motion of a twodimensional Jeffry fluid. Authors in [19-22] studied more properties in Jeffrey fluid. Different non-Newtonian fluids were considered in studies by Pandey and Tripathi [23-25] and Tripathi [26].

Interest in boundary layer flow and heat transfer over a stretching sheet has gained considerable attention because of its application in industry and manufacturing processes. Such applications include polymer extrusion drawing of copper wires, continuous stretching of plastic films and artificial fibers, hot rolling, wire drawing, glass fiber, metal extrusion, and metal spinning. For example, Liu and Andersson [27] studied the heat transfer in a liquid film on an unsteady stretching sheet. The effects of variable fluid properties and thermocapillarity on the flow of a thin film on an unsteady stretching sheet were studied by Dandapat et al. [28]. Hayat et al. [29] investigated the peristaltic mechanism of a Jeffrey fluid in a circular tube. Nadeem et al. [30] analyzed the boundary layer flow of a Jeffrey fluid over an exponentially stretching surface. The effects of thermal radiation are carried out for two cases of heat transfer analysis known as (1) prescribed exponential order surface temperature (PEST) and (2) prescribed exponential order heat flux (PEHF). Hamad [31] studied the convective flow and heat transfer of an incompressible viscous nanofluid past a semi-infinite vertical stretching sheet in the presence of a magnetic field. Hamad and Pop [32] studied theoretically the steady boundary layer flow near the stagnation-point flow on a permeable stretching sheet in a porous medium saturated with a nanofluid and in the presence of internal heat generation/absorption.

The objective of the present study is to investigate the dynamics of the thermal boundary layer flow of a viscous incompressible Jeffrey fluid near the stagnation point on a stretching sheet taking into account the thermal jump condition at the surface. Thus, the main focus of the analysis is to investigate how the flow field, temperature field, shear stress, and heat flux vary within the boundary layer with thermal jump at the wall when the thermal conductivity is temperature dependent. The similarity equations are derived and solved numerically with the widely used and robust computer algebra software. Graphs and tables are presented to illustrate and discuss important hydrodynamic and thermal features of the flow.

\section{Problem Formulation}

Consider a steady two dimensional flow of an incompressible Jeffrey fluid near the stagnation point on a stretching/shrinking sheet. The thermal conductivity is assumed to be functions of temperature. A thermal jump condition is assumed to occur at the wall. We are considering Cartesian coordinate system in such a way that $x$-axis is taken along the stretching sheet in the direction of the motion and $y$-axis is normal to it. The plate is stretched in the $x$-direction with a velocity $u_{w}=c x$ defined at $y=0$. The flow and heat transfer characteristics under the boundary layer approximations are governed by the following equations:

$$
\begin{gathered}
\frac{\partial u}{\partial x}+\frac{\partial v}{\partial y}=0 \\
u \frac{\partial u}{\partial x}+v \frac{\partial u}{\partial y} \\
=u_{e} \frac{d u_{e}}{d x}+\frac{v}{1+\gamma_{1}}\left[\frac{\partial^{2} u}{\partial y^{2}}\right. \\
+\gamma_{2}\left(u \frac{\partial^{3} u}{\partial x \partial y^{2}}+v \frac{\partial^{3} u}{\partial y^{3}}-\frac{\partial u}{\partial x} \frac{\partial^{2} u}{\partial y^{2}}\right. \\
\left.\left.+\frac{\partial u}{\partial y} \frac{\partial^{2} u}{\partial x \partial y}\right)\right] \\
\rho C_{p}\left(u \frac{\partial T}{\partial x}+v \frac{\partial T}{\partial y}\right)=\frac{\partial}{\partial y}\left(\kappa(T) \frac{\partial T}{\partial y}\right)
\end{gathered}
$$

with the boundary conditions (see Rahman and Eltayeb [13]):

$$
\begin{gathered}
\quad v=v_{w}(x), \quad u=u_{w}(x)=c x, \\
T_{\text {jump }}=T_{f}-T_{w} \\
=\lambda_{1}\left(\frac{2}{\sigma_{T}}-1\right) \frac{2 \gamma}{\gamma+1} \frac{\kappa(T)}{\mu C_{p}} \frac{\partial T}{\partial y} \quad \text { at } y=0, \\
u=u_{e}(x)=a x, \quad \frac{\partial u}{\partial y}=0, \quad T=T_{\infty} \\
\text { as } y \longrightarrow \infty .
\end{gathered}
$$

Here $x$ and $y$ are the Cartesian coordinates along the plate and normal to it, respectively, $u$ and $v$ are the velocity components along $x$-and $y$-axes, $v_{w}(x)$ is the mass transfer velocity with $v_{w}(x)<0$ for suction and $v_{w}(x)>0$ for injection or withdrawal, $T$ is the fluid temperature, $\alpha$ is thermal diffusivity, $\nu$ is the kinematic viscosity, $\gamma_{1}$ is the ratio of relaxation and retardation times, $\gamma_{2}$ is the relaxation time, $\gamma$ is the ratio of specific heats, $\sigma_{T}$ is the thermal accommodation coefficient, $\lambda_{1}$ is the mean free path, $\mu$ is the dynamic viscosity, and $\kappa(T)$ is the thermal conductivity which can be, following Chiam [1], written as

$$
\kappa=\kappa_{\infty}\left(1+\varepsilon \frac{T-T_{\infty}}{T_{w}-T_{\infty}}\right),
$$

where $\varepsilon$ is the thermal conductivity parameter.

We introduce now the following similarity variables:

$$
\begin{gathered}
\psi=\sqrt{\operatorname{av} x f(\eta),} \quad \theta(\eta)=\frac{\left(T-T_{\infty}\right)}{\left(T_{w}-T_{\infty}\right)}, \\
\eta=\sqrt{\frac{a}{v}} y,
\end{gathered}
$$

where $\psi$ is the stream function which is defined in the usual way as $u=\partial \psi / \partial y$ and $v=-\partial \psi / \partial x$. Thus, $v_{w}(x)=-\sqrt{a \nu} s$, where $s$ is the mass transfer parameter with $s>0$ for suction and $s<0$ for injection, respectively. Substituting (5) and 
(6) into (2) and (3), the following set of ordinary differential equations results in

$$
\begin{aligned}
f^{\prime \prime \prime} & +\left(1+\gamma_{1}\right)\left(f f^{\prime \prime}-f^{\prime 2}\right) \\
& +\beta\left(f^{\prime \prime 2}-f f^{\prime \prime \prime \prime \prime}\right)+\left(1+\gamma_{1}\right)=0, \\
& (1+\varepsilon \theta) \theta^{\prime \prime}+\varepsilon \theta^{\prime 2}+\operatorname{Pr} f \theta^{\prime}=0,
\end{aligned}
$$

and the boundary conditions (4) become

$$
\begin{gathered}
f(0)=s, \quad f^{\prime}(0)=\lambda, \\
\theta(0)=1+\frac{2 \gamma T_{s}}{\gamma+1} \operatorname{Pr}(1+\varepsilon \theta(0)) \theta^{\prime}(0), \\
f^{\prime}(\infty)=1, \quad f^{\prime \prime}(\infty)=0, \quad \theta(\infty)=0,
\end{gathered}
$$

where $\operatorname{Pr}=\mu C_{p} / \kappa_{\infty}$ is the Prandtl number, $\lambda=c / a$ is the stretching $(\lambda>0)$ or shrinking $(\lambda<0)$ parameter, $\beta=c \gamma_{2}$ is the Deborah number, $T_{s}=\lambda_{1}\left(2 /\left(\sigma_{T}-1\right)\right) \sqrt{a / \nu}$ is the slip parameter, and primes denote differentiation with respect to $\eta$.

2.1. Particular Case. It is worth mentioning that for a regular viscous fluid ( $\left.\beta=\gamma_{1}=0\right)$, (7) reduce to the steady state equations from the paper by Nazar et al. [33] when we neglect the radiation effect.

2.2. Physical Quantities. The physical quantities of interest are the skin friction coefficient $C_{f}$ and the local Nusselt number $\mathrm{Nu}_{x}$, which are defined as

$$
C_{f}=\frac{\tau_{w}}{\rho u_{e}^{2}(x)}, \quad \mathrm{Nu}_{x}=\frac{x q_{w}}{\kappa\left(T_{w}-T_{\infty}\right)},
$$

where $\tau_{w}$ is the skin friction or shear stress along the stretching surface and $q_{w}$ is the heat flux from the surface, which are given by

$$
\tau_{w}=\mu\left(\frac{\partial u}{\partial y}\right)_{y=0}, \quad q_{w}=-\kappa\left(\frac{\partial T_{\mathrm{jump}}}{\partial y}\right)_{y=0} .
$$

Using (6), we get

$$
\begin{gathered}
\operatorname{Re}_{x}^{1 / 2} C_{f}=f^{\prime \prime}(0), \\
\operatorname{Re}_{x}^{-1 / 2} \mathrm{Nu}_{x} \\
=-\frac{2 \gamma}{\gamma+1} T_{s} \operatorname{Pr}\left[(1+\varepsilon \theta) \theta^{\prime \prime}(0)+\varepsilon\left(\theta^{\prime}(0)\right)^{2}\right],
\end{gathered}
$$

where $\operatorname{Re}_{x}=u_{e}(x) x / v$ is the local Reynolds number.

\section{Results and Discussion}

The transformed equations (7) with boundary conditions (8) are solved numerically by using a finite difference method. The asymptotic boundary conditions at $\eta=\infty$ are replaced by $\eta=6$. In Table 1 , we have shown the variation of wall temperature and heat transfer rates with the Prandtl numbers for three different values of Deborah numbers. It is observed

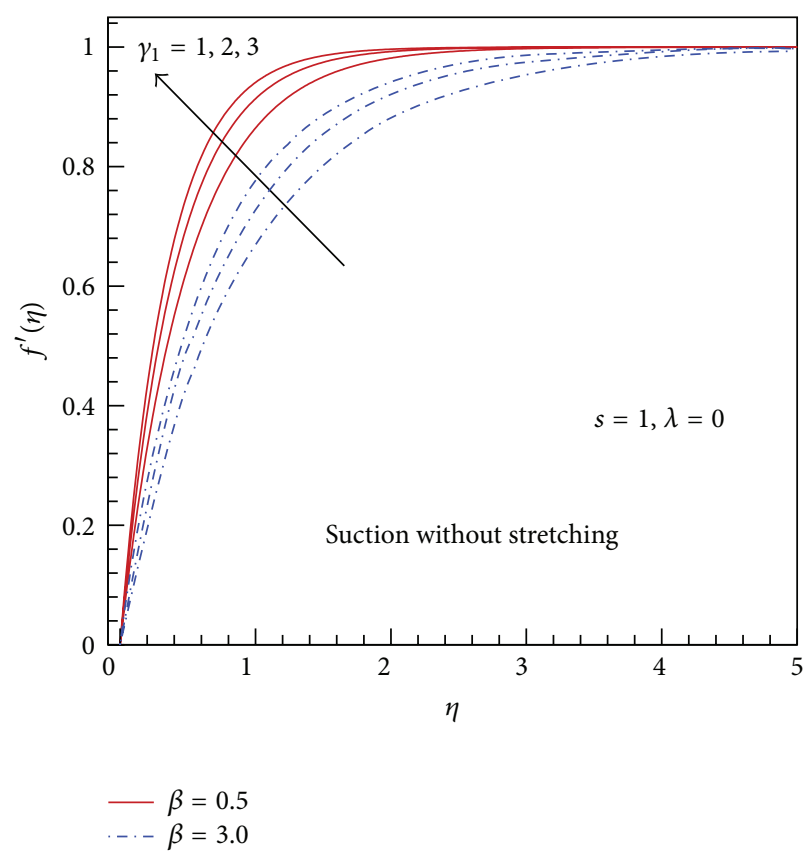

FIGURE 1: Effect of $\beta$ and $\gamma_{1}$ on the velocity profiles.

that the wall temperature decreases whereas the heat transfer rates increase with an increase in Prandtl number. However, the wall temperature increases slightly and heat transfer values decrease slightly with an increase in Deborah number, whereas both decrease with an increase in the ratio of specific heats. This is shown in Tables 1(a) and 1(b). Tables 1(c) and $1(\mathrm{~d})$ show the effects of slip temperature on the wall temperature and heat transfer rates for the constant thermal conductivity. Table 1(e) shows the effects of thermal slip on the wall temperature and heat transfer rates when the thermal conductivity varies with temperature. It can be seen that both the wall temperature and heat transfer rates decrease with an increase in the Prandtl number due to decrease in the thermal conductivity. The effects of the ratio of relaxation and retardation times, Deborah number, and suction and stretching parameters on the $x$-component of velocity are shown in Figures 1 and 2. Figure 1 shows the effects in the absence of stretching. It shows that the velocity increases as the ratio of relaxation and retardation times increases but decreases with an increase in Deborah number. The velocity boundary layer converges quickly for small Deborah numbers. In fact, small Deborah numbers correspond to situations where the material has time to relax (and behaves in a viscous manner), while high Deborah numbers correspond to situations where the material behaves rather elastically. Figure 2 shows the effects of stretching parameter on the velocity for different values of Deborah number. It is observed that the velocity becomes constant when $\lambda=0$, increases when $\lambda<1$, and decreases when $\lambda>0$. Accordingly, the velocity decreases or increases with Deborah number when $\lambda<1$ or $\lambda>0$.

The effect of thermal conductivity parameter on temperature profiles is shown in Figure 3 for two different Prandtl numbers. It is observed that the thermal boundary layer 
TABLE 1: Wall temperature and heat transfer values when one has the following.

(a) $s=1, \lambda=0.5, \gamma_{1}=1, \varepsilon=0, T_{s}=0.1, \gamma=0.5$

\begin{tabular}{|c|c|c|c|c|c|c|}
\hline \multirow{2}{*}{$\operatorname{Pr}$} & \multicolumn{2}{|c|}{$\beta=0.5$} & \multicolumn{2}{|c|}{$\beta=1.0$} & \multicolumn{2}{|c|}{$\beta=1.5$} \\
\hline & $\theta(0)$ & $-\theta^{\prime}(0)$ & $\theta(0)$ & $-\theta^{\prime}(0)$ & $\theta(0)$ & $-\theta^{\prime}(0)$ \\
\hline 1 & 0.9132 & 1.3014 & 0.9139 & 1.2922 & 0.9142 & 1.2865 \\
\hline 2 & 0.7516 & 1.8627 & 0.7526 & 1.8553 & 0.7532 & 1.8509 \\
\hline 3 & 0.5883 & 2.0586 & 0.5892 & 2.0540 & 0.5898 & 2.0512 \\
\hline 4 & 0.4540 & 2.0474 & 0.4547 & 2.0447 & 0.4552 & 2.0431 \\
\hline 5 & 0.3523 & 1.9430 & 0.3529 & 1.9415 & 0.3532 & 1.9405 \\
\hline 6 & 0.2772 & 1.8069 & 0.2776 & 1.8060 & 0.2778 & 1.8055 \\
\hline 7 & 0.2218 & 1.6676 & 0.2221 & 1.6670 & 0.2222 & 1.6667 \\
\hline 8 & 0.1804 & 1.5368 & 0.1806 & 1.5364 & 0.1807 & 1.5362 \\
\hline 9 & 0.1490 & 1.4184 & 0.1491 & 1.4181 & 0.1492 & 1.4180 \\
\hline 10 & 0.1248 & 1.3128 & 0.1249 & 1.3127 & 0.1250 & 1.3126 \\
\hline
\end{tabular}

(b) $s=1, \lambda=0.5, \gamma_{1}=1, \varepsilon=0, T_{s}=0.1, \gamma=1.0$

\begin{tabular}{|c|c|c|c|c|c|c|}
\hline \multirow{2}{*}{$\operatorname{Pr}$} & \multicolumn{2}{|c|}{$\beta=0.5$} & \multicolumn{2}{|c|}{$\beta=1.0$} & \multicolumn{2}{|c|}{$\beta=1.5$} \\
\hline & $\theta(0)$ & $-\theta^{\prime}(0)$ & $\theta(0)$ & $-\theta^{\prime}(0)$ & $\theta(0)$ & $-\theta^{\prime}(0)$ \\
\hline 1 & 0.8753 & 1.2473 & 0.8761 & 1.2389 & 0.8766 & 1.2336 \\
\hline 2 & 0.6686 & 1.6570 & 0.6698 & 1.6511 & 0.6705 & 1.6476 \\
\hline 3 & 0.4878 & 1.7072 & 0.4888 & 1.7040 & 0.4894 & 1.7021 \\
\hline 4 & 0.3567 & 1.6084 & 0.3573 & 1.6067 & 0.3577 & 1.6057 \\
\hline 5 & 0.2661 & 1.4677 & 0.2666 & 1.4668 & 0.2669 & 1.4663 \\
\hline 6 & 0.2036 & 1.3273 & 0.2039 & 1.3268 & 0.2041 & 1.3265 \\
\hline 7 & 0.1597 & 1.2005 & 0.1599 & 1.2002 & 0.1600 & 1.2000 \\
\hline 8 & 0.1279 & 1.0901 & 0.1281 & 1.0899 & 0.1282 & 1.0898 \\
\hline 9 & 0.1045 & 0.9950 & 0.1046 & 0.9949 & 0.1047 & 0.9948 \\
\hline 10 & 0.0868 & 0.9132 & 0.0869 & 0.9131 & 0.0869 & 0.9131 \\
\hline
\end{tabular}

(c) $s=1, \lambda=0.5, \gamma_{1}=1, \varepsilon=0, T_{s}=0.5, \gamma=0.5$

\begin{tabular}{lccccccc}
\hline $\operatorname{Pr}$ & $\beta=0.5$ & \multicolumn{2}{c}{$\beta=1.0$} & \multicolumn{2}{c}{$\beta=1.5$} \\
& $\theta(0)$ & $-\theta^{\prime}(0)$ & $\theta(0)$ & $-\theta^{\prime}(0)$ & $\theta(0)$ & $-\theta^{\prime}(0)$ \\
\hline 1 & 0.6780 & 0.9661 & 0.6797 & 0.9611 & 0.6807 & 0.9579 \\
2 & 0.3771 & 0.9344 & 0.3783 & 0.9326 & 0.3791 & 0.9314 \\
3 & 0.2223 & 0.7778 & 0.2229 & 0.7771 & 0.1432 & 0.7767 \\
4 & 0.1426 & 0.6431 & 0.1430 & 0.6428 & 0.0984 & 0.5426 \\
5 & 0.0981 & 0.5411 & 0.0983 & 0.5410 & 0.0714 & 0.4643 \\
6 & 0.0713 & 0.4644 & 0.0714 & 0.4643 & 0.0541 & 0.4054 \\
7 & 0.0539 & 0.4055 & 0.0540 & 0.4054 & 0.0422 & 0.3592 \\
8 & 0.0422 & 0.3592 & 0.0422 & 0.3592 & 0.0339 & 0.3220 \\
9 & 0.0338 & 0.3221 & 0.0339 & 0.3220 & 0.0278 & 0.2917 \\
10 & 0.0277 & 0.2917 & 0.0278 & 0.2917 & & \\
\hline
\end{tabular}

(d) $s=1, \lambda=0.5, \gamma_{1}=1, \varepsilon=0, T_{s}=0.5, \gamma=1$

\begin{tabular}{lcrccrr}
\hline $\operatorname{Pr}$ & $\beta=0.5$ & \multicolumn{2}{c}{$\beta=1.0$} & \multicolumn{2}{c}{$\beta=1.5$} \\
\hline 1 & $\theta(0)$ & $-\theta^{\prime}(0)$ & $\theta(0)$ & $-\theta^{\prime}(0)$ & $\theta(0)$ & $-\theta^{\prime}(0)$ \\
\hline 2 & 0.5839 & 0.8321 & 0.5858 & 0.8284 & 0.5870 & 0.8260 \\
3 & 0.2875 & 0.7125 & 0.2886 & 0.7114 & 0.1609 & 0.7108 \\
4 & 0.1600 & 0.5600 & 0.1605 & 0.5596 & 0.1002 & 0.4499 \\
5 & 0.0998 & 0.4501 & 0.1001 & 0.4500 & 0.0679 & 0.3729 \\
6 & 0.0676 & 0.3730 & 0.0678 & 0.3729 & 0.0488 & 0.3171 \\
\hline
\end{tabular}


(d) Continued.

\begin{tabular}{lcrrrrrr}
\hline $\operatorname{Pr}$ & $\beta=0.5$ & & & \multicolumn{2}{c}{$\beta=1.5$} \\
& $\theta(0)$ & & $-\theta^{\prime}(0)$ & $\theta(0)$ & $-\theta^{\prime}(0)$ & $\theta(0)$ & $-\theta^{\prime}(0)$ \\
\hline 7 & 0.0366 & 0.2753 & 0.0367 & 0.2752 & 0.0367 & 0.2752 \\
8 & 0.0285 & 0.2429 & 0.0285 & 0.2429 & 0.0286 & 0.2429 \\
9 & 0.0228 & 0.2172 & 0.0228 & 0.2172 & 0.0229 & 0.2171 \\
10 & 0.0187 & 0.1963 & 0.0187 & 0.1963 & 0.0187 & 0.1963 \\
\hline
\end{tabular}

(e) $s=1, \lambda=0.5, \gamma_{1}=1, \varepsilon=-0.5, T_{s}=0.5, \gamma=0.5$

\begin{tabular}{|c|c|c|c|c|c|c|}
\hline \multirow{2}{*}{$\operatorname{Pr}$} & \multicolumn{2}{|c|}{$\beta=0.5$} & \multicolumn{2}{|c|}{$\beta=1.0$} & \multicolumn{2}{|c|}{$\beta=1.5$} \\
\hline & $\theta(0)$ & $-\theta^{\prime}(0)$ & $\theta(0)$ & $-\theta^{\prime}(0)$ & $\theta(0)$ & $-\theta^{\prime}(0)$ \\
\hline 1 & 0.6884 & 1.4256 & 0.6899 & 1.4204 & 0.6908 & 0.6884 \\
\hline 2 & 0.3812 & 1.1468 & 0.3824 & 1.1455 & 0.3831 & 0.3812 \\
\hline 3 & 0.2236 & 0.8741 & 0.2242 & 0.8737 & 0.2246 & 0.2236 \\
\hline 4 & 0.1431 & 0.6922 & 0.1434 & 0.6921 & 0.1436 & 0.1431 \\
\hline 5 & 0.0983 & 0.5690 & 0.0985 & 0.5689 & 0.0986 & 0.0983 \\
\hline 6 & 0.0713 & 0.4815 & 0.0715 & 0.4815 & 0.0715 & 0.0713 \\
\hline 7 & 0.0540 & 0.4167 & 0.0541 & 0.4167 & 0.0541 & 0.0540 \\
\hline 8 & 0.0422 & 0.3669 & 0.0422 & 0.3669 & 0.0423 & 0.0422 \\
\hline 9 & 0.0338 & 0.3276 & 0.0339 & 0.3276 & 0.0339 & 0.0338 \\
\hline 10 & 0.0277 & 0.2958 & 0.0278 & 0.2958 & 0.0278 & 0.0277 \\
\hline
\end{tabular}

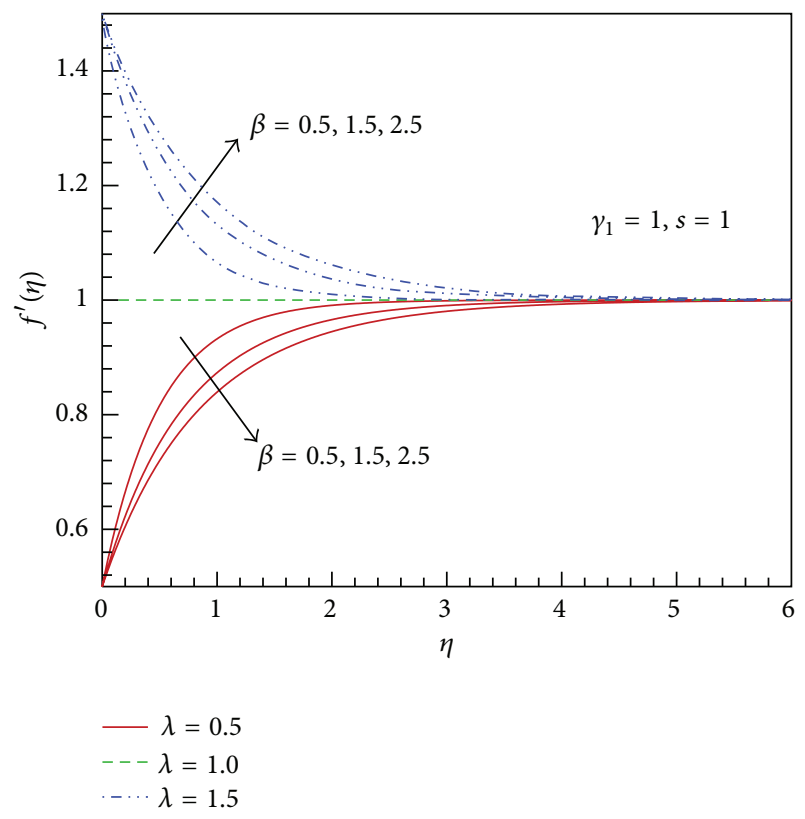

Figure 2: Effect of $\beta$ and $\lambda$ on the velocity profiles.

thickness decreases with an increase in Prandtl number. As the thermal conductivity parameter increases, the temperature in the thermal boundary layer increases. The variaton of skin friction with the ratio of relaxation and retardation times for different parameters is shown in Figures 4(a) and 4(b). When there is no stretching, the skin friction increases with the ratio of relaxation and retardation times and decreases with an increase in Deborah number. As expected, the skin friction reduces with an increase in the suction parameter in both cases. Comparing Figures 4(a) and 4(b), it can be

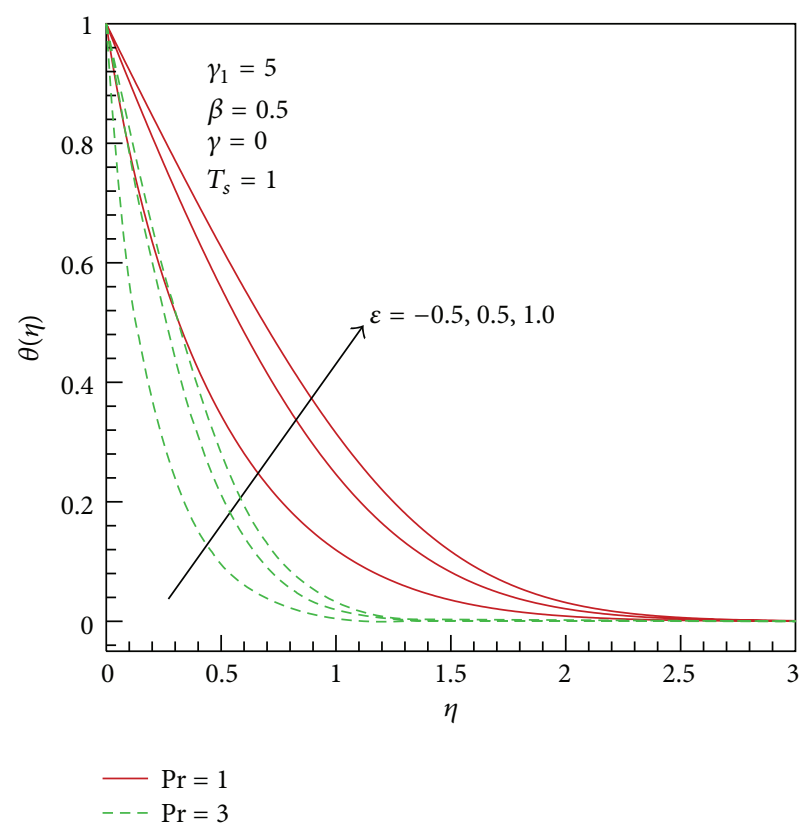

FIgURE 3: Effect of $\operatorname{Pr}$ and $\varepsilon$ on the temperature profiles.

seen that the skin friction decreases with an increase in the stretching parameter. The variation in heat transfer rates with the ratio of relaxation and retardation times is shown in Figures 5 and 6 for different values of suction and thermal conductivity parameters and Prandtl and Deborah numbers. The other parameters are kept constant. As the ratio of relaxation and retardation times increases, the heat transfer rate increases. For higher values of the suction parameter, the heat transfer rates are found to be higher. It is also observed that the heat transfer rates decrease with an increase in 


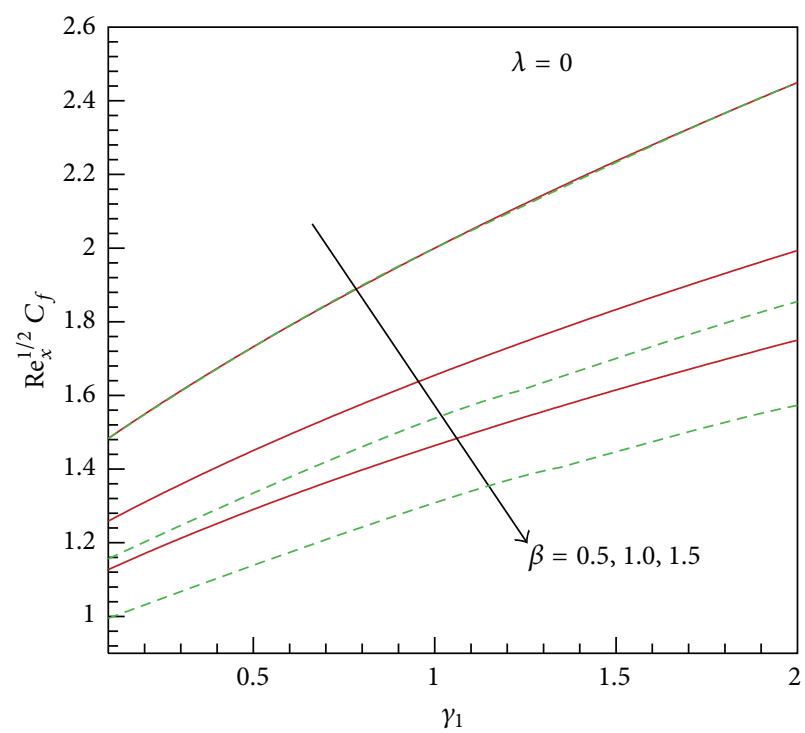

$-s=1$

$--s=3$

(a)

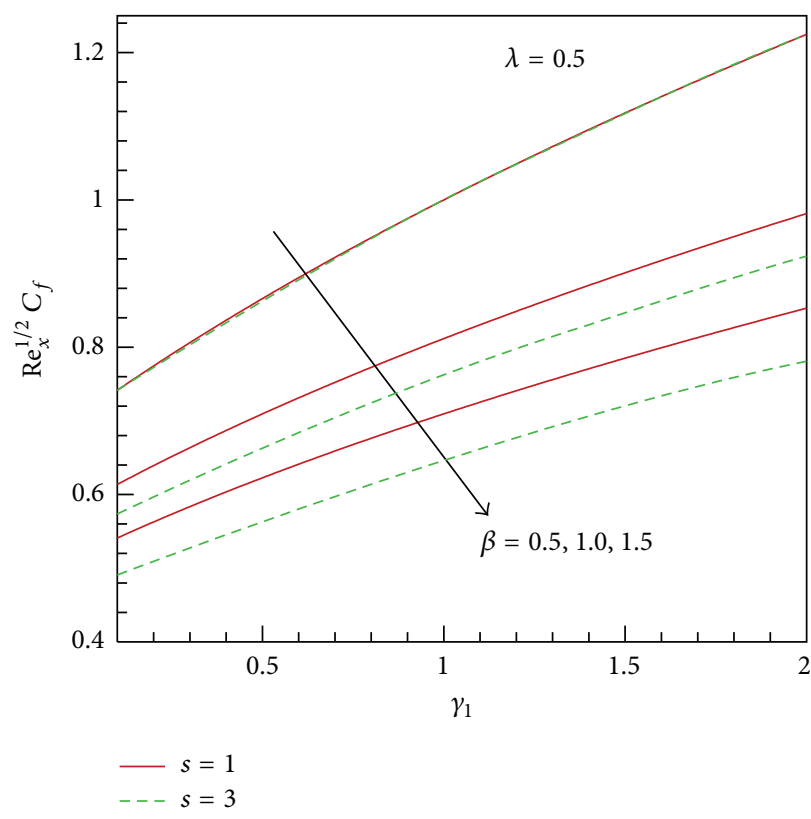

(b)

Figure 4: Effect of $\beta$ and $\lambda$ on the skin friction profiles.

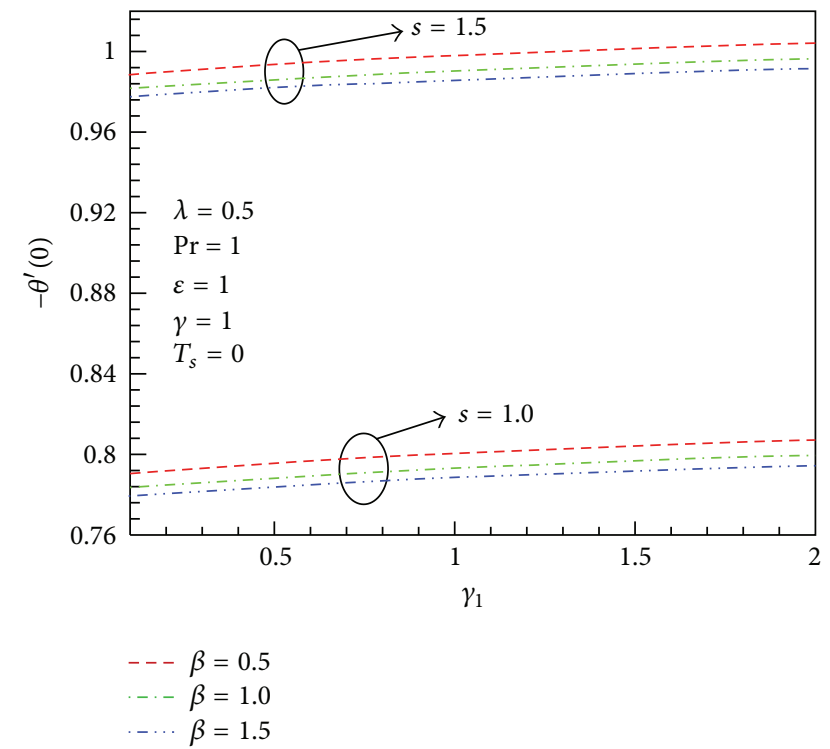

FIGURE 5: Effect of $\beta$ and $s$ on the heat transfer rate.

Deborah number. This is shown in Figure 5. As evident from Figure 6, the heat transfer rates increase with an increase in the Prandtl number. Figure 6 also shows that the heat transfer rates decrease with an increase in the thermal conductivity parameter.

\section{Conclusions}

The effects of thermal jump and variable thermal conductivity on flow and heat transfer near the stagnation point on a

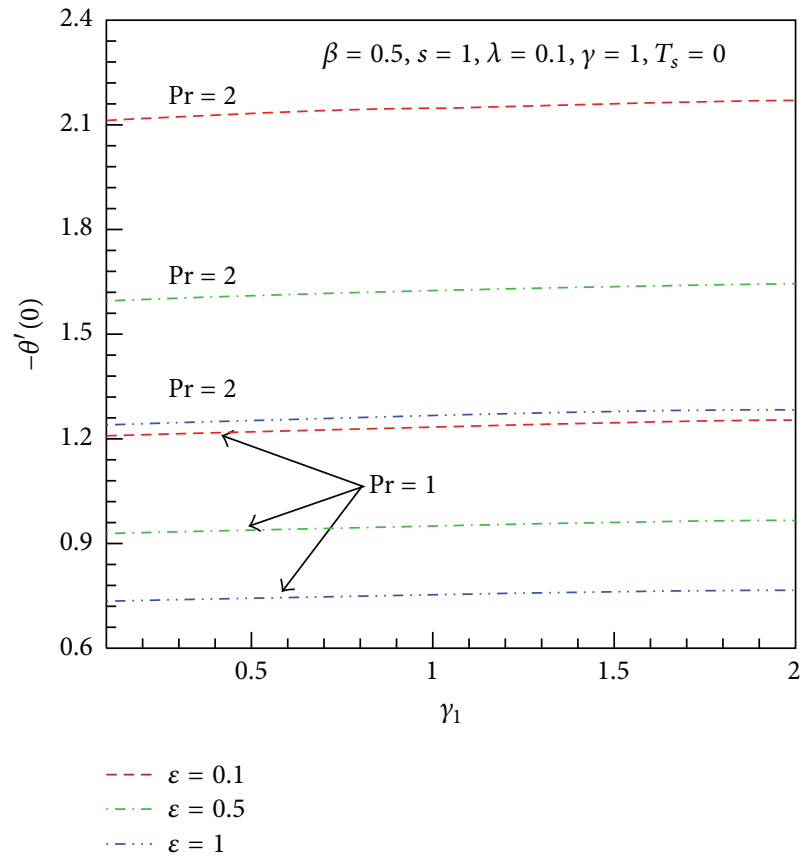

Figure 6: Effect of $\operatorname{Pr}$ and $\varepsilon$ on the heat transfer rate.

stretching/shrinking sheet are investigated numerically in a Jeffrey fluid. The effects of governing parameters including ratio of relaxation and retardation times $\gamma_{1}$, Deborah number $\beta$, Prandtl number, stretching parameter $\lambda$, suction parameter $s$, and thermal conductivity parameter $\varepsilon$ on the dimensionless velocity, temperature, skin friction, and heat transfer rates are investigated and are presented graphically and in tabular form. We conclude the following. 
(a) The wall temperature increases slightly while heat transfer values decrease slightly with an increase in Deborah number.

(b) The wall temperature and heat transfer decrease with an increase in the ratio of specific heats.

(c) The decreases of Deborah number and the increases of relaxation and retardation times leads to increases in the velocity.

(d) The skin friction decreases with an increase in the stretching parameter.

(e) As the ratio of relaxation and retardation times increases, the heat transfer rate increases.

(f) For higher values of the suction parameter, the heat transfer rates are found to be higher.

(g) The heat transfer rates decrease with an increase in Deborah number.

\section{References}

[1] T. C. Chiam, "Heat transfer with variable conductivity in a stagnation-point flow towards a stretching sheet," International Communications in Heat and Mass Transfer, vol. 23, no. 2, pp. 239-248, 1996.

[2] T. C. Chiam, "Heat transfer in a fluid with variable thermal conductivity over a linearly stretching sheet," Acta Mechanica, vol. 129, no. 1-2, pp. 63-72, 1998.

[3] P. S. Datti, K. V. Prasad, M. S. Abel, and A. Joshi, "MHD viscoelastic fluid flow over a non-isothermal stretching sheet," International Journal of Engineering Science, vol. 42, no. 8-9, pp. 935946, 2004.

[4] K. V. Prasad, M. S. Abel, and S. K. Khan, "Momentum and heat transfer in visco-elastic fluid flow in a porous medium over a non-isothermal stretching sheet," International Journal of Numerical Methods for Heat and Fluid Flow, vol. 10, no. 8, pp. 786$801,2000$.

[5] M. S. Abel, K. V. Prasad, and A. Mahaboob, "Buoyancy force and thermal radiation effects in MHD boundary layer visco-elastic fluid flow over continuously moving stretching surface," International Journal of Thermal Sciences, vol. 44, no. 5, pp. 465-476, 2005.

[6] K. V. Prasad and K. Vajravelu, "Heat transfer in the MHD flow of a power law fluid over a non-isothermal stretching sheet," International Journal of Heat and Mass Transfer, vol. 52, no. 2122, pp. 4956-4965, 2009.

[7] M. S. Abel, P. G. Siddheshwar, and N. Mahesha, "Effects of thermal buoyancy and variable thermal conductivity on the MHD flow and heat transfer in a power-law fluid past a vertical stretching sheet in the presence of a non-uniform heat source," International Journal of Non-Linear Mechanics, vol. 44, no. 1, pp. $1-12,2009$.

[8] M. A. Seddeek, S. N. Odda, and M. S. Abdelmeguid, "Numerical study for the effects of thermophoresis and variable thermal conductivity on heat and mass transfer over an accelerating surface with heat source," Computational Materials Science, vol. 47, no. 1, pp. 93-98, 2009.

[9] K. V. Prasad, D. Pal, V. Umesh, and N. S. P. Rao, "The effect of variable viscosity on MHD viscoelastic fluid flow and heat transfer over a stretching sheet," Communications in Nonlinear
Science and Numerical Simulation, vol. 15, no. 2, pp. 331-344, 2010.

[10] M. S. Abel, P. G. Siddheshwar, and N. Mahesha, "Numerical solution of the momentum and heat transfer equations for a hydromagnetic flow due to a stretching sheet of a non-uniform property micropolar liquid," Applied Mathematics and Computation, vol. 217, no. 12, pp. 5895-5909, 2011.

[11] N. C. Mahanti and P. Gaur, "Effects of varying viscosity and thermal conductivity on steady free convective flow and heat transfer along an isothermal vertical plate in the presence of heat sink," Journal of Applied Fluid Mechanics, vol. 2, no. 1, pp. 23-28, 2009.

[12] R. G. Deissler, "An analysis of second-order slip flow and temperature-jump boundary conditions for rarefied gases," International Journal of Heat and Mass Transfer, vol. 7, no. 6, pp. 681694, 1964.

[13] M. M. Rahman and I. A. Eltayeb, "Convective slip flow of rarefied fluids over a wedge with thermal jump and variable transport properties," International Journal of Thermal Sciences, vol. 50, no. 4, pp. 468-479, 2011.

[14] J. W. Cipolla Jr., "Heat transfer and temperature jump in a polyatomic gas," International Journal of Heat and Mass Transfer, vol. 14, no. 10, pp. 1599-1610, 1971.

[15] T.-T. Kao, "Laminar free convective heat transfer response along a vertical flat plate with step jump in surface temperature," Letters in Heat and Mass Transfer, vol. 2, no. 5, pp. 419-428, 1975.

[16] A. V. Latyshev and A. A. Yushkanov, "An analytic solution of the problem of the temperature jumps and vapour density over a surface when there is a temperature gradient," Journal of Applied Mathematics and Mechanics, vol. 58, no. 2, pp. 259-265, 1994.

[17] M. Turkyilmazoglu and I. Pop, "Exact analytical solution for the flow and heat transfer near the stagnation point on a stretching/shrinking sheet in a Jeffrey fluid," International Journal of Heat and Mass Transfer, vol. 57, no. 1, pp. 82-88, 2013.

[18] S. Akram and S. Nadeem, "Influence of induced magnetic field and heat transfer on the peristaltic motion of Jeffrey fluid in an asymmetric channel: closed form solutions," Journal of Magnetism and Magnetic Materials, vol. 328, pp. 11-20, 2013.

[19] C. E. Siewert and D. Valougeorgis, "The temperature-jump problem for a mixture of two gases," Journal of Quantitative Spectroscopy and Radiative Transfer, vol. 70, no. 3, pp. 307-319, 2001.

[20] S. Nadeem, A. Hussain, and M. Khan, "Stagnation flow of a Jeffrey fluid over a shrinking sheet," Zeitschrift fur Naturforschung A, vol. 65, no. 6-7, pp. 540-548, 2010.

[21] S. K. Pandey and D. Tripathi, "Unsteady model of transportation of Jeffrey-fluid by peristalsis," International Journal of Biomathematics, vol. 3, no. 4, pp. 473-491, 2010.

[22] T. Hayat, M. Awais, S. Asghar, and A. A. Hendi, "Analytic solution for the magnetohydrodynamic rotating flow of Jeffrey fluid in a channel," Journal of Fluids Engineering, vol. 133, no. 6, Article ID 061201, 2011.

[23] S. K. Pandey and D. Tripathi, "Influence of magnetic field on the peristaltic flow of a viscous fluid through a finite-length cylindrical tube," Applied Bionics and Biomechanics, vol. 7, no. 3, pp. 169-176, 2010.

[24] S. K. Pandey and D. Tripathi, "Effects of non-integral number of peristaltic waves transporting couple stress fluids in finite length channels," Zeitschrift fur Naturforschung A, vol. 66, no. 3-4, pp. 172-180, 2011.

[25] S. K. Pandey and D. Tripathi, "Unsteady peristaltic flow of micro-polar fluid in a finite channel," Zeitschrift fur Naturforschung A, vol. 66, no. 3-4, pp. 181-192, 2011. 
[26] D. Tripathi, "A mathematical model for the peristaltic flow of chyme movement in small intestine," Mathematical Biosciences, vol. 233, no. 2, pp. 90-97, 2011.

[27] I.-C. Liu and H. I. Andersson, "Heat transfer in a liquid film on an unsteady stretching sheet," International Journal of Thermal Sciences, vol. 47, no. 6, pp. 766-772, 2008.

[28] B. S. Dandapat, B. Santra, and K. Vajravelu, "The effects of varable fluid properties and thermocapillarity on the flow of a thin film on an unsteady stretching sheet," International Journal of Heat and Mass Transfer, vol. 50, no. 5-6, pp. 991-996, 2007.

[29] T. Hayat, N. Ali, and S. Asghar, "An analysis of peristaltic transport for flow of a Jeffrey fluid," Acta Mechanica, vol. 193, no. 1-2, pp. 101-112, 2007.

[30] S. Nadeem, S. Zaheer, and T. Fang, "Effects of thermal radiation on the boundary layer flow of a Jeffrey fluid over an exponentially stretching surface," Numerical Algorithms, vol. 57, no. 2, pp. 187-205, 2011.

[31] M. A. A. Hamad, "Analytical solution of natural convection flow of a nanofluid over a linearly stretching sheet in the presence of magnetic field," International Communications in Heat and Mass Transfer, vol. 38, no. 4, pp. 487-492, 2011.

[32] M. A. A. Hamad and I. Pop, "Scaling Transformations for Boundary Layer Flow near the Stagnation-Point on a Heated Permeable Stretching Surface in a Porous Medium Saturated with a Nanofluid and Heat Generation/Absorption Effects," Transport in Porous Media, vol. 87, no. 1, pp. 25-39, 2011.

[33] R. Nazar, N. Amin, D. Filip, and I. Pop, "Unsteady boundary layer flow in the region of the stagnation point on a stretching sheet," International Journal of Engineering Science, vol. 42, no. 11-12, pp. 1241-1253, 2004. 

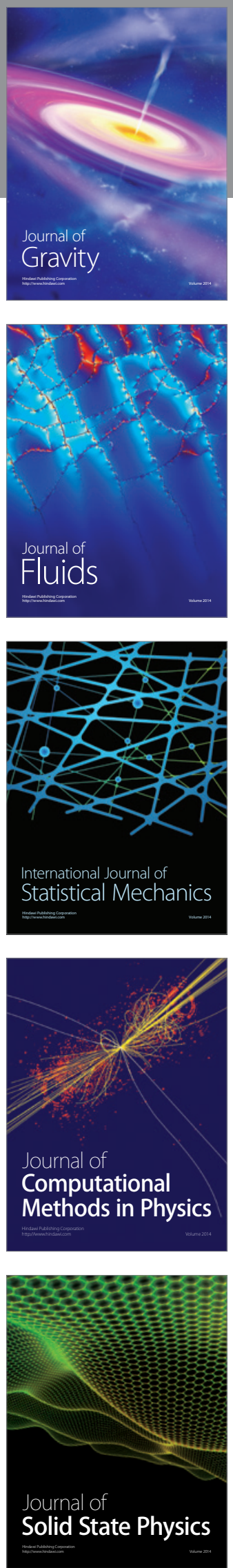

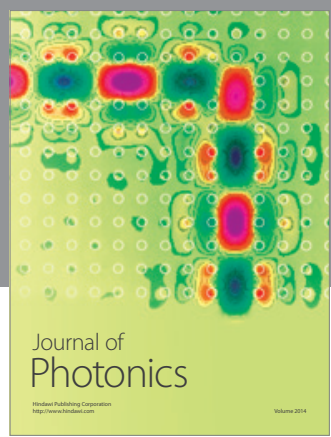

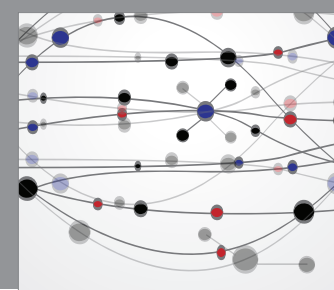

The Scientific World Journal

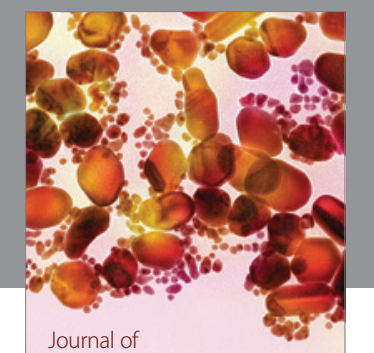

Soft Matter
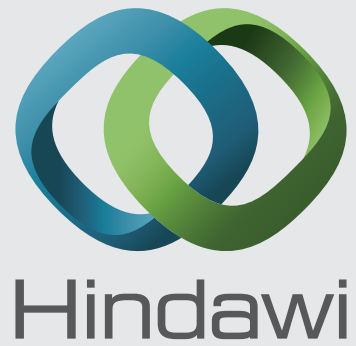

Submit your manuscripts at

http://www.hindawi.com
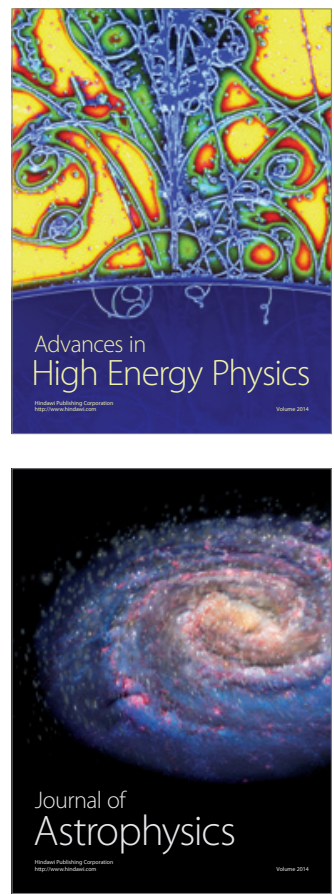
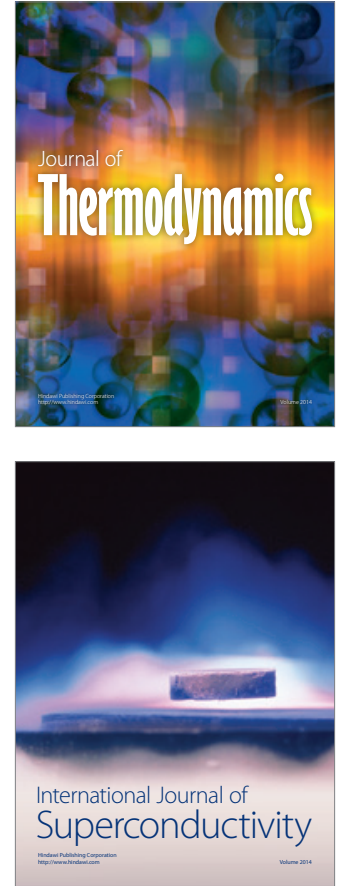
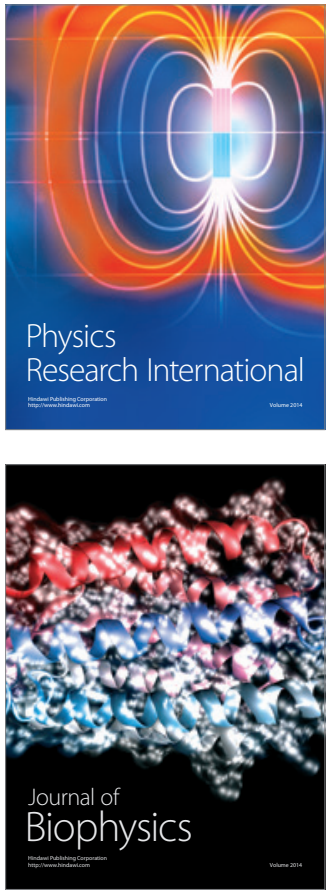
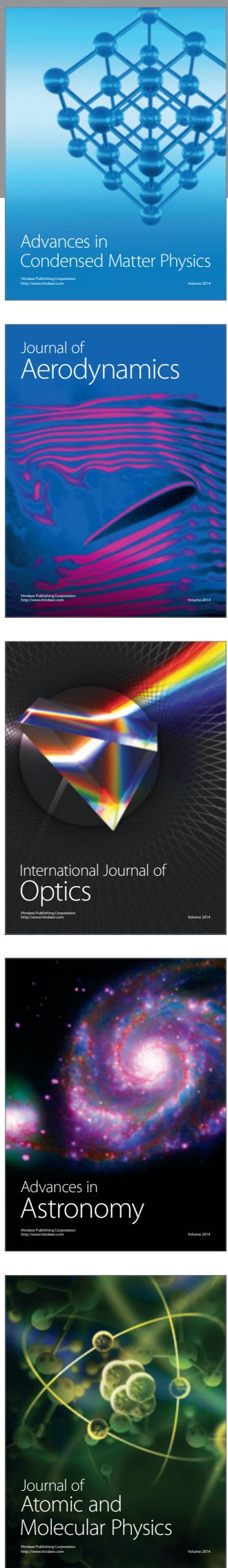